Conclusion/next steps PLHIV possesses the social capital needed to build working-networks with government and non-government organisations to increase the quality of their lives, therefore shouldn't be objectified by any party whether a project, a government or an-international institution.

\section{P14.11 DEVELOPING SUSTAINABLE, INTERNATIONAL PARTNERSHIPS MODEL TO BUILD CAPACITY IN HIV AND STIS}

'S Sawleshwarkar*, ${ }^{2}$ A Singh, ${ }^{2}$ S Zodpey, ${ }^{1} R J$ Hillman. 'Western Sydney Sexual Health Centre, University of Sydney, Sydney, Australia; ${ }^{2}$ Public Health Foundation of India, New Delhi, India

\subsection{6/sextrans-2015-052270.523}

Introduction Effective public health responses in prevention and management of HIV/STIs require strong human resources, focused on the development and implementation of evidencebased policies. We wished to explore how best to leverage Australian pedagogic and research skills in HIV/STIs into an Indian setting, in order to build human resource capacity.

Methods An appropriate institutional partner in India was chosen on the basis of their academic track record. Financial support was obtained from the Australian Government's aid agency. Three phases were developed:

Phase 1: Stakeholder consultation and survey to establish local needs, together with pedagogic skill development at the partner institution;

Phase 2: Curriculum mapping and development to reflect local educational priorities;

Phase 3: Pilot delivery, followed by regional expansion.

Results The University of Sydney partnered with the Public Health Foundation of India (PHFI), due to their focus on public health education, coupled with their technical and pedagogic expertise. 27 local faculty members of PHFI and affiliated organisations were trained in Australia and an enabling environment created by reciprocal visits and close collaboration. Forty three stakeholders from 14 Indian institutions participated in the national consultation. These included academic institutions, the National AIDS Control Organisation, NGOs and prospective participants. The preference was for a 6-month course focussing on public health aspects of HIV/STIs.

To minimise costs, an e-learning site was developed using open source software and containing jointly developed content. The pilot course was delivered in 2013-2014, subsequently extended to include a regional component. A total of 53 students have successfully completed the course to date.

Conclusion Clarity of purpose, careful matching of institutions and effective communication were keys to building informed partnerships. The leveraging of existing expertise, local adaptation, together with the use of open-source software has led to the development and running of a cost effective, scalable capacity-building resource.

Disclosure of interest statement Australian aid agency of the Department of Foreign Affairs and Trade, Australia funded the project.

\section{P14.12 NSW SEXUAL HEALTH PROMOTION}

${ }^{1} \mathrm{D}$ Welsby*, ${ }^{1} \mathrm{~J}$ Holden, ${ }^{2} \mathrm{~J}$ De Wit. ${ }^{1}$ Centre for Population Health, NSW Ministry of Health, Australia; ${ }^{2}$ Centre for Social Research in Health, University of NSW, Australia

\subsection{6/sextrans-2015-052270.524}

Background Sexual health promotion is central to the prevention of human immunodeficiency virus (HIV) and sexually transmissible infections (STIs) and to achieving the targets of the NSW HIV Strategy 2012-2015 and the forthcoming NSW STI Plan.

In NSW sexual health promotion targeting young people is delivered by local health district HIV and Related Programs (HARP) Units, non-government organisations and state-wide services. In the past state level leadership and coordination of sexual health promotion has been limited, consequently many services have been working in isolation and delivering small scale programs with limited evaluation.

Methods During 2014 the NSW Ministry of Health (MOH) undertook a review with the aim of strengthening the State's response to sexual health promotion. The review involved a staged approach including:

1. An evidence check of evidence-based practice in HIV, STI and viral hepatitis health promotion and Aboriginal community engagement;

2. A review of data;

3. Consultation with key stakeholders to map existing infrastructure and resources, current sexual health promotion work and to identify barriers and enablers for the services.

Results The evidence check found patchy evidence to guide health promotion efforts. The review of current sexual health promotion across NSW identified a gap in state-wide prevention activity targeting young people aged 16 to 29 was. Young people have high notification rates for chlamydia, high rates of partner change and barriers to accessing health services.

Conclusions In response to these findings $\mathrm{MOH}$ developed the NSW Sexual Health Promotion Framework for the delivery of an integrated population-based prevention program that aims to increase consistent condom use and health seeking behaviours (STI/HIV testing and treatment) among young people. The Framework also aims to reduce duplication, strengthen partnerships across the sector and enable robust evaluation and monitoring of program outcomes. Implementation of the Framework has commenced.

Disclosure of interest statement Nil.

\section{P14.13 HIV-RELATED STIGMA AND UNIVERSAL TESTING AND TREATMENT FOR HIV PREVENTION AND CARE: DESIGN OF AN IMPLEMENTATION SCIENCE EVALUATION NESTED IN THE HPTN 071 (POPART) CLUSTER- RANDOMISED TRIAL IN ZAMBIA AND SOUTH AFRICA}

${ }^{1} \mathrm{JR}$ Hargreaves*, ${ }^{2} \mathrm{~A}$ Stangl, ${ }^{1,3} \mathrm{~V}$ Bond, ${ }^{4} \mathrm{G}$ Hoddinott, ${ }^{1} \mathrm{~S}$ Krishnaratne, ${ }^{4} \mathrm{H}$ Mathema, ${ }^{3} \mathrm{M}$ Moyo, ${ }^{4} \mathrm{~L}$ Viljoen, ${ }^{2} \mathrm{~L}$ Brady, ${ }^{2} \mathrm{~K}$ Siewwright, ${ }^{4} \mathrm{~L}$ Horn, ${ }^{1} \mathrm{~K}$ Sabapathy, ${ }^{1,3} \mathrm{H}$ Ayles, ${ }^{4} \mathrm{~N}$ Beyers, ${ }^{4} \mathrm{P}$ Bock, ${ }^{5} \mathrm{~S}$ Fidler, ${ }^{6} \mathrm{~S}$ Griffith, ${ }^{1} \mathrm{~J}$ Seeley, ${ }^{1} \mathrm{R}$ and Hayes, on behalf of the HPTN 071 (PopART) study team. 'London School of Hygiene and Tropical Medicine, Keppel Street, London, WC1E 7HT, UK; ${ }^{2}$ International Center for Research on Women, 1120 20th St NW, Suite 500 North, Washington, DC, 20036, USA; ${ }^{3}$ Zambart, ZAMBART House, Ridgeway Campus, Lusaka, Zambia; ${ }^{4}$ Desmond Tutu TB Centre, Department of Paediatrics and Child Health, Stellenbosch University, Lower Level Clinical Building, Fransie Van Zyl Street, Parow Valley, Cape Town, 7500; ${ }^{5}$ Imperial College London, SW7 2AZ, UK; ${ }^{6}$ FHI 360, 359 Blackwell Street, Suite 200, Durham, NC 27701, USA

10.1136/sextrans-2015-052270.525 
Background Stigma and discrimination related to HIV and populations at high risk of HIV have the potential to impede the implementation of effective HIV prevention and treatment programmes. We will conduct an implementation science study of HIV-related stigma in communities and health settings within a large, pragmatic cluster-randomised trial of a universal testing and treatment intervention for HIV prevention in Zambia and South Africa and assess how stigma affects, and is affected by, implementation of this intervention.

Methods A mixed-method evaluation will be nested within HPTN071/PopART (Clinical Trials registration number NCT01900977), a three-arm trial comparing universal door-todoor delivery of HIV testing and referral to prevention and treatment services, accompanied by either an immediate offer of antiretroviral treatment to people living with HIV (PLHIV) regardless of clinical status, or an offer of treatment in-line with national guidelines, with a standard-of-care control arm. The primary outcome of HPTN071/PopART is HIV incidence measured among a cohort of 52,500 individuals in 21 study clusters. Our evaluation will include integrated quantitative and qualitative data collection and analysis in all sites. We will collect quantitative data on indicators of HIV-related stigma over three years from large probability samples of community members, health workers, and PLHIV, and qualitative data, including in-depth interviews and observations from members of these same groups sampled purposively. In analysis we will: (i) compare HIV-related stigma measures between study arms, (ii) link data on stigma to measures of the success of implementation of the intervention, (iii) explore changes in the drivers and manifestations of stigma in study communities and the health system.

Discussion Using a novel study-design nested within a large, pragmatic trial we will evaluate the extent to which HIV-related stigma affects and is affected by the implementation of a comprehensive combination HIV prevention intervention including a universal test and treatment approach.

Disclosure of interest statement HPTN 071 is sponsored by the National Institute of Allergy and Infectious Diseases (NIAID) under Cooperative Agreements UM1-AI068619, UM1AI068617, and UM1-AI068613, with funding from the US President's Emergency Plan for AIDS Relief (PEPFAR). Additional funding is provided by the International Initiative for Impact Evaluation (3ie) with support from the Bill and Melinda Gates Foundation, as well as by NIAID, the National Institute on Drug Abuse (NIDA) and the National Institute of Mental Health (NIMH), all part of NIH. The content is solely the responsibility of the authors and does not necessarily represent the official views of the NIAID, NIMH, NIDA, PEPFAR, 3ie, or the Bill and Melinda Gates Foundation. We have no conflicts of interest to declare.

\section{P14.14 INTERSECTING STIGMAS: A FRAMEWORK FOR DATA COLLECTION AND ANALYSIS OF STIGMAS FACED BY PEOPLE LIVING WITH HIV AND KEY POPULATIONS}

${ }^{1} \mathrm{JR}$ Hargreaves*, ${ }^{2} \mathrm{~A}$ Stangl, ${ }^{1,3} \mathrm{~V}$ Bond, ${ }^{4} \mathrm{G}$ Hoddinott, ${ }^{1} \mathrm{~S}$ Krishnaratne, ${ }^{4} \mathrm{H}$ Mathema ${ }^{3} \mathrm{M}$ Moyo, on behalf of the HPTN071 (PopART) Study Team. 'London School of Hygiene and Tropical Medicine; ${ }^{2}$ International Center for Research on Women; ${ }^{3}$ ZAMBART Project; ${ }^{4}$ Desmond Tutu Tuberculosis Centre, Stellenbosch University

\subsection{6/sextrans-2015-052270.526}

Introduction Stigma can impede the implementation of HIV prevention and treatment programmes. Many measurement scales have been developed, though few have addressed intersecting stigmas: that is to say, multiple stigmas faced by people living with HIV, and key populations at high risk of HIV.

Methods We developed an approach to measure intersecting stigmas nested within the HPTN 071 (PopART) trial, conducted in South Africa and Zambia. We adopted best-practice wording to assess key domains of stigma, building on a process of global consultative indicator harmonisation. We designed nested data collection items in surveys for parallel use among the general population, health workers and people living with HIV (PLHIV) - including health workers living with HIV - to assess the same phenomena from multiple perspectives. We also designed "parallel" assessments of key population stigma within the health care worker survey to compare the attitudes and perceptions of health care workers towards PLHIV, and those at increased risk of HIV infection, such as migrants, sex workers, MSM, young women, and people with disabilities.

A core set of seven questions was included in the HPTN071 (PopART) surveys conducted among the general population (over 10,000 individuals), PLHIV (about 4000 individuals) and health care workers (over 1000 individuals). These items assessed two key domains of HIV stigma: drivers of stigma and manifestations of stigma. In the health care worker survey, questions specific to key populations were also included, to assess health care worker attitudes, experiences and perceptions about those at increased risk of HIV as listed above. We developed an approach to visually represent the complex data set using methods based on social-network analysis.

We developed an approach to assess intersecting HIV-related stigmas, incorporating data collected from different population groups. Data collection is ongoing.

Disclosure of interest statement HPTN 071 is sponsored by the National Institute of Allergy and Infectious Diseases (NIAID) under Cooperative Agreements UM1-AI068619, UM1AI068617, and UM1-AI068613, with funding from the US President's Emergency Plan for AIDS Relief (PEPFAR). Additional funding is provided by the International Initiative for Impact Evaluation (3ie) with support from the Bill and Melinda Gates Foundation, as well as by NIAID, the National Institute on Drug Abuse (NIDA) and the National Institute of Mental Health (NIMH), all part of NIH. The content is solely the responsibility of the authors and does not necessarily represent the official views of the NIAID, NIMH, NIDA, PEPFAR, 3ie, or the Bill and Melinda Gates Foundation. We have no conflicts of interest to declare.

\section{P14.15 IN THE SYSTEM (TONGAN SYSTEM) BUT OUT OF PLACE}

Joey Joleen Siosaia*. WCA Mataele - Tonga Leitis' Association/Pacific Sexual Diversity Network

\subsection{6/sextrans-2015-052270.527}

Introduction Since HIV/AIDS was discovered in the early 80 s, and the first AIDS Victim came to Tonga from the United States, 19 people have lost their lives to the virus leaving many children and MSM/TG with more discriminations for our Communities. Since then there has been a lot of Stigma and Discriminations on our young MSM/TGs. These children have been forced out of school, engaged in hard labour, prostitution or high risk behaviours that make them vulnerable to contracting HIV. With support from international donors and local resource efforts.

Methods Tonga Leiti's Association (TLA) has a long and complex history. It's a history of survival. Its history has been 